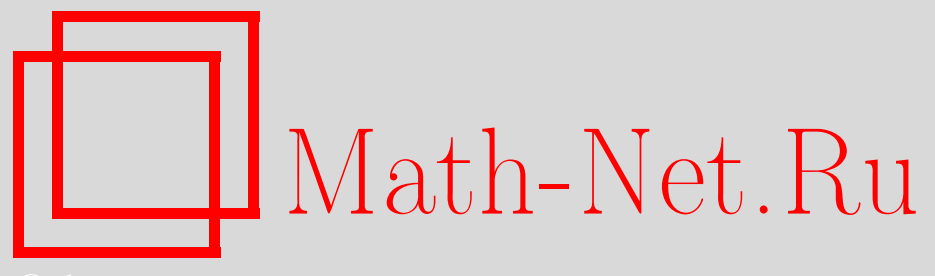

А. В. Тимофеенко, О порождающих тройках инволюций больших спорадических групп, Дискрет. матем., 2003, том 15, выпуск 2, 103-112

DOI: https://doi.org/10.4213/dm197

Использование Общероссийского математического портала Math-Net.Ru подразумевает, что вы прочитали и согласны с пользовательским соглашением http://www.mathnet.ru/rus/agreement

Параметры загрузки:

IP: 52.23 .180 .231

26 апреля 2023 г., 03:54:37

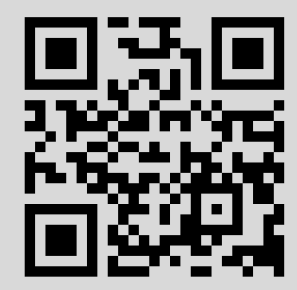




\title{
О порождающих тройках инволюций больших спорадических групп
}

\author{
(c) 2003 г. А. В. Тимофеенко
}

За исключением групп Бэби-Монстр $B$, Монстр $M$, группы Маклафлина $M c L$ и групп Матье $M_{11}, M_{22}, M_{23}$ в каждой конечной простой спорадической группе указаны порождающие ее три инволюции, две из которых перестановочны. Если $G$ - одна из групп $M_{12}, M_{24}, H S, J_{1}, J_{2}, J_{3}$, то найдены все такие пары чисел $p, q, p \leqslant q$, что $p=|i k|, q=|j k|$ для некоторых порождающих группу $G$ инволюций $i, j, k$ с условием $|i j|=2$.

Указанные выше тройки инволюций обнаружены с помощью системы компьютерной алгебры GAP. Напомним, что в тройке инволюций, которые порождают одну из групп $M c L, M_{11}, M_{22}, M_{23}$, любые две инволюции не перестановочны.

Работа выполнена при поддержке Российского фонда фундаментальных исследований, проект 02-01-00078.

\section{1. Введение}

Напомним [1], что к настоящему времени все конечные простые группы описаны. Они разделены на бесконечные серии знакопеременных групп и групп лиева типа, а также на серию из 26 исключительных (спорадических) групп. Большинство спорадических групп открыто сравнительно недавно (после 1964 г.) и требует объяснения своей исключительности. Беспрецедентность объема текста классификационного доказательства (до 20000 журнальных страниц) выдвигает на первый план поиск нового доказательства, а также изучение самих конечных простых групп. В частности, с 1980 г. в Коуровской тетради [2] стонт проблема В. Д. Мазурова (проблема 7.30):

какие конечные простые группы порождаются тремя инволюциями, две из которых перестановочны?

Для бесконечных серий простых конечных групп ответ на этот вопрос нашел Я. Н. Нужин в цикле работ (см. [3] и библиографию в ней). Ответ оказался отрицательным только для знакопеременных групп $A_{6}, A_{7}, A_{8}$ и ряда линейных групп размерностей, меньших 5. В случаях положительного ответа искомые инволюции указаны в явном виде. Кроме того, Я. Н. Нужин поставил более общий вопрос:

какие конечные простые группы являются гомоморфными образами группы Кокстера

$$
\operatorname{gr}\left(a, b, c \mid a^{2}, b^{2}, c^{2}, a c a c,(a b)^{p},(b c)^{q}\right)
$$

при фиксированных показателях $p$ и $q$, особенно для $p, q \leqslant 6 ?$ 
Известно, что проблемой В. Д. Мазурова для спорадических групп занимался Б. Л. Абашеев [2]. Он доказал существование требуемых инволюций в каждой спорадической группе за исключением групा

$$
M_{11}, \quad M_{22}, \quad M_{23}, \quad M c L
$$

и группы Монстр $M$. Для последней группы положительное решение проблемы В. Д. Мазурова нашел С. Нортон [2]. Результаты Б. Л. Абашеева и С. Нортона не опубликованы, а из сообщений в [2] следует, что существование в группе порождающих ее трех инволюций, две из которых перестановочны, доказано ими без явного построения самих инволюций.

В работе [4] автора доказано, что в тройке инволюций, которые порождают группу списка (1), любые два элемента не перестановочны. А для 14 спорадических групп там явно указаны порождающие их тройки инволюций, две из которых коммутируют.

В настоящей работе аналогичный результат получен еще для шести спорадических групाп

$$
J_{4}, \quad C o_{1}, \quad F i_{24}^{\prime}, \quad H N, \quad T h, \quad L y .
$$

Как и в [4], порождающие тройки инволющий строятся с помощью системы компьютерной алгебры GAP (см. http : / /www-gap.dcs . st-and.ac . uk/ gap). Однако высокая степень подстановочного либо матричного представления каждой из этих групп потребовала создания новых алгоритмов поиска инволюций. Некоторые из них анонсированы в $[5,6]$.

Таким образом, вопрос о явном указании нужных инволюций остается открытым только для самых больших (и по количеству элементов и по степени представления) спорадических групп-монстров $B$ и $M$. Компьютерные вычисления даже в группе БэбиМонстр $B$ упираются в значительные затраты времени.

Теорема 1. За исключением групп Бэби-Монстр В, Монстр M, группы Маклафлина McL и групn Mатье $M_{11}, M_{22}, M_{23}$ в каждой конечной простой спорадической группе указаны порождающие ее три инволючии, две из которых перестановочны. Более того, если $G$ - одна из групn

$$
M_{12}, \quad M_{24}, \quad H S, \quad J_{1}, \quad J_{2}, \quad J_{3},
$$

то найдены все пары $(p, q)$ чисел $p, q, p \leqslant q$, такие, что $p=|i k|, q=|j k|$ для некоторых порождающих группу $G$ инволючий $i, j, k$ с условием $|i j|=2$.

Отметим также, что интерес к порождающим тройкам инволюций в конечных простых группах вызван и запросами приложений. Одним из них является построение однородных графов (в которых любые две вершины инцидентны равному числу ребер). Указанные в теореме инволюции помещены в виде подстановок и матриц на сайте http: // icm. krasn. ru 22 августа 2000 г. как файл стандартного GAP-формата. 


\section{2. Схема доказательства теоремы}

Будем пользоваться терминологией и обозначениями из [7].

Автор искал нужные для теоремы инволюции с помощью системы компьютерной алгебры GAP. Отсутствующие в этой системе спорадические группы можно найти в электронном атласе Вилсона представлений конечных групп (см. Wilson R., http: //www.mat.bham.ac.uk/atlas/).

Для каждой группы $G$ определим множество

$$
C_{2}(G)=\{(|a c|,|c b|)|G=\operatorname{gr}(a, b, c),| a|=| b|=| c|=| a b|=2,| a c|\leqslant| c b \mid\} .
$$

Например, для группы $G$ списка (1) множество $C_{2}(G)$ пустое, а если

$$
(p, q) \in\{(3,3),(3,4),(3,6),(4,4)\}, \quad(p, q) \in C_{2}(G),
$$

то группа $G$ не является простой.

Для доказательства теоремы достаточно построить для каждой группы $G$ из (3) множество $C_{2}(G)$ и найти в 20 спорадических простых конечных группах порождающие их тройки инволюций, две из которых перестановочны. Поскольку эти тройки расположены на упоминавшемся выше сайте Красноярского алгебраического семинара (см. http://icm.krasn.ru/seminar/seminar.html), остается проверить перестановочность двух инволюций в каждой из этих троек и выяснить, будет ли такая тройка инволюций порождать соответствующую группу.

Доказательство теоремы фактически ведется отдельно для каждой спорадической группы. С другой стороны, удалось обойтись по-существу тремя алгоритмами. Разобьем 20 нужных нам групп теоремы на три подмножества и для каждого подмножества опишем алгоритм поиска порождающих группу троек инволюций.

Первое подмножество состоит из групп

$$
\left\{M_{12}, M_{24}, H S, J_{1}, J_{2}, J_{3}\right\} \text {. }
$$

Для каждой такой группы $G$ строим все классы еe сопряженных инволюций. В группе $G$ их не более двух. Затем с точностью до сопряженности перебираем все тройки инволюций, сохраняя те из них, которые порождают исследуемую группу и дают новые пары для множества $C_{2}(G)$. Увеличению скорости вычислений служит отказ от попадающих в максимальную подгруппу троек инволюций. Таким перебором было выяснено [4], что группы списка (1) не порождаются тремя инволюциями, две из которых перестановочны.

Второе подмножество состоит из групп

$$
\left\{O N, \mathrm{Fi}_{23}, \mathrm{Fi}_{24}^{\prime}, \mathrm{HN}, \mathrm{Co}_{3}, \mathrm{He}, \mathrm{Ru}, \mathrm{Suz}, \mathrm{Co}_{1}, \mathrm{Co}_{2}, \mathrm{~F}_{22}\right\}
$$

(в построении множества $H N$ принимал участие А. И. Макосий, а множества $R u-$ Е. И. Кузнецов).

В каждой группе $G$ из данного множества инволюции находятся с помощью случайного выбора в классе сопряженных инволюций либо группы, либо ее максимальной подгруппы небольшого порядка. Проверка на порождаемость группы $G$ найденной тройкой инволюций происходит без участия теоретико-вероятностных алгоритмов.

Третъе множество состоит из групп

$$
\left\{J_{4}, L y, T h\right\} \text {. }
$$


В этих группах напрямую не удается проверить, порождают ли три найденные инволющии соответствующую спорадическую группу $G$. Приходится опираться на описание ее максимальных подгрупп. В одной из них так выбираются инволюции $i, j$, что в максимальных подгруппах группы $G$, содержащих элементы порядка $|i j|$ нет элементов некоторого порядка $n$. Тогда ищем такую инволюцию $k$, что группа $\operatorname{gr}(i, j, k)$ обладает элементом порядка $n$. Следовательно, $\operatorname{gr}(i, j, k)=G$, и если $k i=i k$ или $j k=k j$, то $i, j, k-$ искомая тройка инволюций.

Для групा

$$
M_{12}, M_{24}, H S, J_{1}, J_{2}, C o_{2}, C o_{3}, F_{22}, F i_{23}, R u, O N, H e, S u z
$$

теорема доказана в [4].

После публикации [4] список спорадических групп, в которых указаны три порождающие их инволюции, пополнен группами (2), поэтому именно для них перечисленные выше алгоритмы и будут описаны подробнее.

Доказательство теоремы начнем с группы $J_{3}$.

\section{3. Третья группа Янко $J_{3}$}

Порождающие группу $J_{3}$ подстановки $a, b$ берем из электронного атласа Вилсона. В виде групповых слов алфавита $\{a, b\}$ этот атлас содержит порождающие элементы максимальной в $J_{3}$ подгруппы $H_{1}$. Строим множество $B$ всех инволюций группы $H_{1}$. Оказалось, что $|B|=323$. Поскольку все инволюции группы $J_{3}$ сопряжены и $|a|=2$, множество $C$ всех инволюций группы $J_{3}$ получаем из класса $a^{J_{3}}$ ее сопряженных инволюций. Переставим элементы множества $C$ так, чтобы первые ее 323 элемента составляли множество $B$. Теперь фиксируем первую в $C$ инволюцию $i$. Вторая, обозначим ее через $j$, пробегает все инволюции из $C$, начиная со второй и заканчивая предпоследней. Для каждой такой инволюции $j$ находим порядок $|i j|$ и выбор третьей инволюцин $k$ осуществляем так. Сначала берем в качестве $k$ следующую за $j$ инволюцию в $C$, а если $j \in B$, то $k$ - 324-я инволюция списка $B$. Затем находим порядки $|i k|,|j k|$. Если оба эти числа и число $|i j|$ отличны от двойки, то в качестве $k$ берем следующую в списке $C$ инволюцию. Если двойка встречается, то составляем пары порядков произведений - кандидаты для попадания в множество $C_{2}\left(J_{3}\right)$. Наконец, если среди этих кандидатов хотя бы один еще не был обнаружен, то проверяем справедливость равенства $|\operatorname{gr}(i, j, k)|=\left|J_{3}\right|$. Если ответ положительный, то $i, j, k$ - искомые инволюции, а в множестве $C_{2}\left(J_{3}\right)$ указаны неизвестные элементы (один, два или три). Оказалось, что

$$
\begin{aligned}
& C_{2}\left(J_{3}\right)=\{(3,10), \quad(3,12), \quad(3,15), \quad(3,17), \quad(4,9), \quad(4,10), \quad(4,15) \text {, } \\
& (5,6), \quad(5,8), \quad(5,9),(5,10),(5,12),(5,15), \quad(5,17) \text {, } \\
& (6,6),(6,8),(6,9),(6,10),(6,12),(6,15),(6,17) \text {, } \\
& (8,9),(8,10),(8,12),(8,15),(8,17) \text {, } \\
& (9,9), \quad(9,10), \quad(9,12), \quad(9,15), \quad(9,17) \text {, } \\
& (10,10),(10,12),(10,15),(10,17) \text {, } \\
& (12,12),(12,15),(12,17),(15,15),(15,17),(17,17)\} \text {. }
\end{aligned}
$$




\section{4. Четвертая группа Янко $J_{4}$}

Как известно (см., например, электронный атлас Вилсона), в группе $J_{4}$ представитель только одного класса сопряженных максимальных подгрупп обладает элементом порядка 43. Он является группой Фробениуса $H_{12}=(e) \lambda(f)$, причем $|e|=43,|f|=14$. В ее диэдральной подгруппе $\operatorname{gr}\left(e, f^{7}\right)$ берем инволюции $i=f^{7}$ и $j=e i$. Понятно, что $|i j|=43$, и если в централизаторе $C_{J_{4}}(i)$ взять инволюцию $k, k \neq i, \operatorname{to} \operatorname{gr}(i, j, k)=J_{4}$. Итак, доказано существование в группе $J_{4}$ трех порождающих ее инволюций, две из которых перестановочны.

Опишем теперь алгоритм построения элементов $i, j, k$. Будем применять обозначения из атласа Вилсона, где матрицы $a$ и $b$ размерности $112 \times 112$ над полем $G F(2)$ названы стандартными порождающими группы $J_{4}$. Там же находим порождающие ее максимальную подгруппу $H_{12}$ элементы $h_{1}$ и $h_{2}$ в виде групповых слов в алфавите $\{a, b\}$. Теперь подключаем систему компьютерной алгебры GAP. Оказывается, что $\left|h_{1} h_{2}\right|=14,\left|h_{1}\right|=2$. Возьмем $i=\left(h_{2} h_{1}\right)^{7}, j=h_{1}$ и проверим справедливость равенства $|i j|=43$.

Строить централизатор $C_{J_{4}}(i)$ - задача неподъемная для доступных автору компьютеров. Поэтому находим все инволюции в какой-нибудь небольшой подгруппе и проверяем, нет ли среди них перестановочных с инволюцией $i$. В качестве небольшой подгруппы в атласе Вилсона берем десятую в порядке убывания порядков максимальную подгруппу $H_{10}$ группы $J_{4}$. Подгруппа $H_{10}$ задана там своими порождающими словами $h_{10}^{\prime}$ и $h_{10}^{\prime \prime}$ в алфавите $\{a, b\}$, то есть матрицами. Попытаемся с помощью системы GAP найти в $H_{10}$ все перестановочные с $i$ инволюции.

Поскольку $H_{10} \simeq P S U_{3}(9)$, удобно пользоваться точным подстановочным представлением 28-й степени группы $P_{3} U_{3}(9)$. Оно требует значительно меньших ресурсов машины, чем 112-мерное линейное представление. Становится возможным для каждой инволюции $k_{10}$ из $H_{10}$ найти ее запись групповым словом в алфавите $\left\{h_{10}^{\prime}, h_{10}^{\prime \prime}\right\}$. Точнее, на базе подстановочного представления 28-й степени группы $H_{10}$ система GAP строит еще одно представление группы $H_{10}$. Композиция двух этих точных представлений отображает матрицы $h_{10}^{\prime}$ и $h_{10}^{\prime \prime}$ в абстрактные символы $x_{1}$ и $x_{2}$, а каждую инволюцию $k_{10}$ из $C_{H_{10}}(i)$ - в групповое слово в алфавите $\left\{x_{1}, x_{2}\right\}$. Теперь символам $x_{1}$ и $x_{2}$ присваиваем матричные значения $h_{10}^{\prime}$ и $h_{10}^{\prime \prime}$ соответственно, а считанная заново запись инволюции $k_{10}$ произведением элементов $x_{1}$ и $x_{2}$ дает $k_{10}$ в виде 112-мерной матрицы.

Вычислим порядок $\left|i k_{10}\right|$. Оказалось, что все такие порядки четные, но ни один из них не равен 2. Однако, каждая инволюция $\left(i k_{10}\right)^{\left|i k_{10}\right| / 2}$, обозначим ее посредством $k$, перестановочна с $i$ и отлична от $i$. Порождающая групту $J_{4}$ тройка $(i, j, k)$ инволюций, две из которых ( $i$ и $k$ ) перестановочны, построена.

Громоздкость матриц $i, j, k$ не позволяет привести их здесь. Они могут быть найдены на упоминавшемся сайте Красноярского алгебраического семинара. С другой стороны, инволюции $i, j, k$ представлены ниже произведениями порождающих группу $J_{4}$ стандартных матриц $a$ и $b$ из электронного атласа Вилсона:

$$
\begin{aligned}
& j=\left(\left((a b)^{3} b b\right)^{6}\right)^{a b a b\left((a b)^{3} b a b b\right)^{26},} \\
& i=\left(\left(\left((a b a b b)^{2} a b^{3}\right)^{3}\right)^{\left.\left((a b)^{3} b a b b\right)^{33}(a b)^{22} j\right)^{7},}\right. \\
& k=\left(i a^{b(b a)^{8}(a b)^{18}}\right)^{8} .
\end{aligned}
$$


Пусть

$$
\begin{aligned}
& x=a^{b(b a)^{8}(a b)^{18}}, \\
& y=(b a)^{-24}(a b)^{16}(a b a b a b b)^{4}(a b)^{-16}(b a)^{24}, \\
& z=\left(\left(\left(a b a b b a b a b b a b^{3}\right)^{3}\right)^{\left.\left((a b)^{3} b a b b\right)^{33}(a b)^{22} j\right)^{7},}\right.
\end{aligned}
$$

где $a$ и $b$ - стандартные порождающие группы $J_{4}$. Каждое из следующих произведений матриц $x, y$ и $z$ можно взять в качестве инволющии $k$ в порождающей группу $J_{4}$ тройке инволюций $i, j, k$, две из которых перестановочны:

$$
\begin{aligned}
& \left(x y x y^{4} x y^{2} x y^{5} x z\right)^{11}, \quad(x z)^{8}, \quad\left(x y x y x y x y x y^{2} x y z\right)^{12}, \\
& \left(y^{4} x y x y x y^{2} x y x y x y^{3} z\right)^{12}, \quad\left(y x y^{3} x y^{2} z\right)^{10} \text {, } \\
& \left(y x y x y x y x y^{2} x y x z\right)^{11}, \quad\left(x y x y^{3} x y^{2} x z\right)^{8} \text {, } \\
& \left(y x y^{4} x y^{2} x y^{5} z\right)^{8}, \quad\left(x y x y x y x y^{4} x y^{4} x y x y z\right)^{8}, \quad\left(x y^{5} x y x z\right)^{10} \text {, } \\
& \left(y x y^{2} x y^{4} x y^{5} z\right)^{12}, \quad\left(y^{5} x y z\right)^{10}, \quad\left(y x y^{2} x y^{4} x y^{2} x y^{3} z\right)^{6}, \\
& \left(x y x y x y x y^{2} x y x y z\right)^{6}, \quad\left(x y^{2} x y^{3} x y x z\right)^{11} \text {, } \\
& \left(y x y^{2} x y^{2} x y x y^{2} x y^{2} x z\right)^{8}, \quad\left(y^{5} x y x y x y^{2} x y x y x y^{2} z\right)^{5} \text {, } \\
& \left(y x y x y x y^{2} x y x y x z\right)^{8}, \quad\left(y^{2} x y^{3} x y z\right)^{11}, \quad\left(y^{3} z\right)^{8} \text {, } \\
& \left(y x y^{3} x y^{5} z\right)^{11}, \quad\left(y^{2} x y x y x y^{2} x y x y x y^{5} z\right)^{8} \text {, } \\
& \left(x y x y^{2} x y^{2} x y x y^{2} x y^{2} z\right)^{12}, \quad\left(x y x y x y^{4} x y^{4} x y x y x y z\right)^{5} \text {, } \\
& \left(x y x y x y^{2} x y x y x y z\right)^{11}, \quad\left(y^{3} x y x y^{5} x y^{3} z\right)^{10} \text {, } \\
& \left(x y^{2} x y^{2} x y x y^{2} x y^{2} x y z\right)^{11}, \quad\left(x y^{3} x z\right)^{11} \text {, } \\
& \left(x y x y^{3} x y^{5} x z\right)^{3}, \quad\left(x y x y x y x y x y^{4} x y x y z\right)^{8} \text {, } \\
& \left(x y^{2} x y x y^{2} x y^{2} x y x y^{2} z\right)^{6}, \quad\left(y x y x y^{4} x y^{4} x y x y x y x z\right)^{11} \text {, } \\
& \left(y x y x y^{2} x y x y x y x z\right)^{8}, \quad\left(y x y^{2} x y x y^{2} x y^{2} x y x y z\right)^{12} \text {, } \\
& \left(y^{2} x y x y^{2} x y^{2} x y x y^{2} x z\right)^{8}, \quad\left(x y^{3} x y x y^{2} x y^{3} x y^{3} z\right)^{6} \text {, } \\
& \left(y^{2} x y^{2} x y x y^{2} x y^{2} x y x z\right)^{11}, \quad\left(x y x y^{2} x y x y x y x y z\right)^{8} \text {, } \\
& \left(x y x y x y^{3} x y^{2} x y^{5} x z\right)^{12}, \quad\left(x y^{4} x y^{2} x z\right)^{11} \text {, } \\
& \left(x y x y^{5} x z\right)^{10}, \quad\left(y^{2} x y x y^{3} x y^{2} x y^{4} z\right)^{12}, \quad\left(y^{2} x y^{3} x y^{4} z\right)^{10} \text {, } \\
& \left(x y^{2} x y x y^{2} x y x y x y x y^{5} z\right)^{5}, \quad\left(y^{2} x y x y^{2} x y x y x y x y^{5} z\right)^{12} \text {, } \\
& \left(y x y^{2} x y x y x y x y x z\right)^{11}, \quad\left(y x y x y^{3} x y^{2} x y^{5} z\right)^{12} \text {, } \\
& \left(x y^{2} x y^{4} x y^{2} x y^{4} z\right)^{11}, \quad\left(y^{2} x y^{4} x y^{2} x y^{4} z\right)^{2}, \quad\left(y^{4} x y^{2} z\right)^{10} \text {, } \\
& \left(y x y^{5} z\right)^{11}, \quad\left(y^{2} x y x y^{5} x y^{4} z\right)^{11}, \quad\left(x y^{2} x y^{3} x y^{4} x z\right)^{12} \text {, } \\
& \left(y x y x y^{2} x y^{2} x y x y^{2} x y z\right)^{8}, \quad\left(y^{2} x y^{4} z\right)^{6}, \quad\left(x y^{5} x y^{2} x y^{3} x y x y x z\right)^{6} \text {, } \\
& \left(y x y^{2} x y^{3} x y x y^{5} z\right)^{11}, \quad\left(x y^{3} x y^{3} z\right)^{6}, \quad\left(y^{3} x y^{3} z\right)^{12} \text {, } \\
& \left(y^{4} x y x y^{3} x y^{2} x y^{2} z\right)^{10}, \quad\left(x y^{2} x y^{4} x z\right)^{12}, \quad\left(y^{3} x y^{4} x y^{2} x y^{3} z\right)^{5} \text {. }
\end{aligned}
$$

Вычисление пары $(|i j|,|j k|)$ или $(|i j k|,|j k|)$ для построенных выше инволюций $i, j$, 
$k$ с условиями $J_{4}=\operatorname{gr}(i, j, k), i k=k i$, приводит к следующему включению:

$$
\begin{aligned}
C_{2}\left(J_{4}\right) \supseteq & \{(4,11),(4,43),(8,12),(8,20),(8,30),(8,37),(8,43),(8,66),(10,10), \\
& (10,31),(10,43),(10,44),(10,66),(11,12),(11,43),(12,15), \\
& (12,16),(12,24),(12,30),(12,37),(12,43),(15,20),(15,24), \\
& (15,43),(16,16),(16,22),(16,30),(16,31),(16,33),(16,40), \\
& (16,43),(16,66),(20,20),(20,31),(20,37),(20,43),(20,66), \\
& (22,23),(22,24),(22,29),(22,30),(22,31),(22,37),(22,40), \\
& (22,43),(22,44),(23,43),(24,24),(24,31),(24,33),(24,37), \\
& (24,40),(24,43),(24,66),(29,43),(30,43),(31,37),(31,43), \\
& (33,40),(33,43),(37,43),(40,43),(43,43),(43,44),(43,66)\} .
\end{aligned}
$$

\section{5. Группа Лайенса $L y$}

Как известно (см., например, электронный атлас Вилсона), в группе $L y$ все максимальные подгруппы с элементом порядка 67 сопряжены и являются группой Фробениуса $H_{8}=(e) \lambda(f)$, причем $|e|=67,|f|=22$. В ее диэдральной подгруппе $\operatorname{gr}\left(e, f^{11}\right)$ берем инволюции $i=f^{11}$ и $j=e i$. Понятно, что $|i j|=67$ и если в централизаторе $C_{L y}(i)$ взять инволюцию $k, k \neq i$, то $\operatorname{gr}(i, j, k)=L y$. Итак, доказано существование в группе $L y$ трех порождающих ее инволюций, две из которых перестановочны.

Опишем теперь алгоритм построения элементов $i, j, k$. Будем применять обозначения из электронного атласа Вилсона, где матрищы $a$ и $b$ размерности $111 \times 111$ над полем $G F(5)$ названы стандартными порождающими группы $L y$. Там же находим порождающие ее максимальную подгруппу $H_{8}$ элементы $a_{8}$ и $b_{8}$ в виде групповых слов в алфавите $\{a, b\}$. Теперь подключаем систему компьютерной алгебры GAP. Оказывается, что $\left|b_{8} a_{8}\right|=22$, $\left|a_{8}\right|=2$. Возьмем $i=\left(b_{8} a_{8}\right)^{11}, j=a_{8}$ и проверим справедливость равенства $|i j|=67$.

Поскольку построение централизатора $C_{L y}(i)$ требует больших машинных ресурсов, к успеху в поиске перестановочных с $i$ инволюций приходим так. Пусть $a_{1}$ и $b_{1}-$ порождающие изоморфной группе лиева типа $G_{2}(5)$ максимальной подгруппы $H$ группы Ly. В алфавите $\{x, y\}$ рассмотрим групповые слова $l_{1}, \ldots, l_{252}$. Они являются записями инволюций группы $P S U_{3}(9) \lambda(d)$, где $|d|=2$, в виде произведений порождающих группу $P_{3} U_{3}(9) \lambda(d)$ подстановок $x, y$. Присвоив символам $x, y$ матричные значения $x=a_{1}$, $y=b_{1}$, считываем снова слова $l_{1}, \ldots, l_{252}$, которые после этого становятся 111-мерными матрицами группы $L y$. Для каждого $r=1, \ldots, 252$ вычисляем порядок $\left|i l_{r}\right|$. Если он четен, то строим инволюцию $k=\left(i l_{r}\right)^{\left|i l_{r}\right| / 2}$ и проверяем, во-первых, ее перестановочность с инволюциями $i, j$, во-вторых, лежит ли она вне подгруппы $H_{8}$ группы $L y$. Когда ответ на оба вопроса положительный, получаем $L y=\operatorname{gr}(i, j, k)$, причем две инволюции в этой порождающей тройке перестановочны.

Громоздкость матриц $i, j, k$ не позволяет привести их здесь, они могут быть найдены на упоминавшемся ранее сайте Красноярского алгебраического семинара. С другой стороны, порождающие группу $L y$ инволюции $i, j, k$, две из которых перестановочны, представлены ниже произведениями порождающих группу $L y$ стандартных матриц $a$ и $b$ 
из атласа Вилсона, любые две из этих троек отличаются только инволюцией $k$ :

$$
\begin{aligned}
j & =a^{(a b a b a b b)^{17}(a b b)^{21},} \\
i & =\left((a b a b b a b b b a b b b)^{(a b b)^{16}(a b a b a b b)^{30}} j\right)^{11}, \\
x & =(a b a b b b)^{7} a(a b a b b b)^{-7}, \\
y & =(a b a b a b b)^{-25}(a b a b a b b a b)^{3}(a b a b a b b)^{25}, \\
k \in\{ & \left\{i x^{15}, i\left(x y x y x y^{3} x y x y^{3} x y^{3} x\right)^{12}, i\left(x y x y x y x y^{2} x y x y\right)^{12},\right. \\
& i\left(x y x y x y x y^{2} x y x y x y^{2} x y x y^{2} x y x y\right)^{12}, \\
& i\left(x y x y x y^{2} x y x y x y^{2} x y x y^{2} x y x y x y\right)^{20}, \\
& i\left(y x y x y x y^{2} x y x y x y^{2} x y x y^{2} x y x y x\right)^{21}, \\
& \left.i\left(x y x y x y^{2} x y x y^{2} x y x y x y^{2} x y x y x y\right)^{14}\right\} .
\end{aligned}
$$

Приходим к построенному с помощью перечисленных выше инволюций $i, j, k$ включению

$$
\begin{array}{r}
C_{2}(L y) \supseteq\{(15,30),(15,67),(18,37),(18,67),(21,37),(21,40),(21,67), \\
(24,37),(24,67),(30,67),(37,42),(37,67),(40,67),(42,67)\} .
\end{array}
$$

\section{6. Спорадическая группа Томпсона $T h$}

Как известно $[8,9]$ (см. также электронный атлас Вилсона), в группе Th представители только двух классов сопряженных максимальных подгрупп содержат элементы порядка 19. Оба они, $H_{4}$ и $H_{12}$, то есть четвертый и двенадцатый по невозрастанию порядков, не имеют элементов порядка 13. Поэтому инволютивные матрицы $i, j, k$ группы $T h$ в силу того, что $|i j|=19$ и $\left|(i j)^{6} k\right|=13$, порождают ее. А ввиду равенств $|i k|=2,|j k|=18$, $|j i k|=18$ инволюции $i$ и $k$, а также $i$ и $i k$ перестановочны, причем порядки произведений неперестановочных инволюций в тройках $(i, j, k)$ и $(i, j, i k)$ составляют пары $(18,19)$ и $(18,18)$ соответственно.

Указанные выше порядки элементов 248-мерного линейного представления группы Томпсона над полем из двух элементов найдены в системе компьютерной алгебры GAP. Стандартные порождающие группу Томпсона матрицы обозначены в процитированном уже атласе буквами $a$ и $b$. Оказалось, что $i=a, j=a^{b a b a b b}$. Обе инволюции взяты из максимальной подгруппы $H_{4}=\operatorname{gr}(a, b a b a b b)$, а для построения третьей инволюции $k$ потребовалось довольно длинное произведение сомножителей $i, j$ и еще одной инволюции из максимальной подгруппы $H_{13} \simeq P S L_{3}(3)$ (содержащей элементы 13-го порядка).

Порождающие группу Томпсона $T h$ инволюции $i, j, k$ расположены на сайте Красноярского алгебраического семинара. Вычисление порядков произведений некоторых из них подтверждает следующее включение

$$
C_{2}(T h) \supset\{(18,18),(18,19)\} .
$$




\section{7. Группа Фишера $\mathrm{Fi}_{24}^{\prime}$}

Искомые тройки инволющий строятся ЭВМ с помощью системы компьютерной алгебры GAP. Алгоритм незначительно отличается от применявшегося для спорадических групп с одним классом сопряженных инволюций и высокой степенью подстановочного представления (см. сайт Красноярского алгебраического семинара). Разница в том, что сначала в группе $F i_{24}^{\prime}$ строится централизатор инволюции $i$ из порождающего множества этой группы. Такую систему $\{i, b\}$ порождающих можно найти, например, в атласе Вилсона представлений конечных групп. Остается к инволюции $i$ добавлять инволюцию $j$ из построенного централизатора и в классе сопряженных инволюций группы $F i_{24}^{\prime}$ выбирать еще один элемент $k$ до тех пор, пока порождающий $b$ не будет лежать в $\operatorname{gr}(i, j, k)$.

Если проверку включения $b \in \operatorname{gr}(i, j, k)$ осуществлять только когда в $C_{2}\left(F i_{24}^{\prime}\right)$ не указана хотя бы одна пара из $(|i k|,|j k|),(|i k|,|i j k|),(|j k|,|i j k|)$, то, остановив счет после рассмотрения сотни подряд элементов $k$, не приведших к появлению в $C_{2}\left(F i_{24}^{\prime}\right)$ новых пар, получаем включение

$$
\begin{array}{r}
C_{2}\left(F i_{24}^{\prime}\right) \supseteq\{(4,12),(5,6),(5,8),(5,10),(5,12),(6,6),(6,8), \\
(6,10),(6,12),(8,12),(10,10),(10,12),(12,12)\} .
\end{array}
$$

\section{8. Группа Харады-Нортона $H N$}

За несколько часов компьютер Pentium III, $533 \mathrm{MHz}$, нашел порядок группы ХарадыНортона $H N$. Значит, технические возможности проверки порождаемости этой группы данными элементами (подстановками 1140000-й степени) имеются.

Сначала в самой маленькой максимальной подгруппе $H_{14}$ порядка 47320 группы $H N$ находим подгруппу Клейна порядка 4. Делается это так. Строится силовская 2-подгруппа группы $H_{14}$, составляется список ее инволюций и для каждой из них вычисляется порядок класса сопряженных с ней элементов. Выбрав представители классов попарно различных порядков, получаем список всех инволюций в виде объединения самих классов. Одну из инволюций этого списка обозначаем буквой $i$. Затем перебираем другие до тех пор, пока не встретится такая инволюция $j$, что $i j=j i$.

Третью нужную нам инволюцию $k$ будем искать в классе $c^{H_{12}}$ сопряженных инволюций другой максимальной в группе $H N$ подгруппы $H_{12}$. Она изоморфна расширению группы Матье $M_{12}$ посредством группы второго порядка. В атласе Вилсона находим порождающие $c$ и $d$ подгруппы $H_{12}$. Оказалось, что $|c|=2$. Инволюция $k$ выбирается в классе $c^{H_{12}}$ случайным образом и на каждом шаге проверяется равенство $\operatorname{gr}(i, j, k)=H N$. Второй шаг оказался результативным, причем

$$
C_{2}(H N) \supset\{(10,10),(10,12)\} .
$$




\section{9. Группа Конвея $\mathrm{Co}_{1}$}

В атласе Вилсона представлений конечных групп нет подстановок, порождающих группу $\mathrm{Co}_{1}$. Однако она изоморфна фактор-групше групшы $\mathrm{Co}_{0}$ по ее центру порядка 2, а группа $\mathrm{Co}_{0}$ порождена в атласе Вилсона перестановками степени 196560. Система GAP вычисляет центр $C$ группы $C o_{0}$ и строит саму группу $C o_{1}$ как фактор-группу $C o_{0} / C$.

Тройку порождающих группу $\mathrm{Co}_{1}$ инволюций $i, j, k$ удобно искать среди прообразов в $C o_{0}$ так же, как это делалось для группы $H N$ и $\mathrm{Fi}_{24}^{\prime}$.

Пусть $C o_{0}=\operatorname{gr}\left(a_{0}, b_{0}\right), C o_{1}=C o_{0} / C$. Будем пользоваться порождающими $a_{0}, b_{0}$ из атласа Вилсона, где $\left|a_{0}\right|=2$. В централизаторе $C_{C o_{0}}\left(a_{0}\right)$ берем инволюцию $j_{0}$. А третью инволюцию $k_{0}$ ищем в классе $a_{0}^{C_{0}}$ сопряженных с ней элементов до тех пор, пока не получим равенства $\operatorname{gr}\left(a_{0}, j_{0}, k_{0}\right)=C o_{0}$. Положим теперь $i=a_{0} C, j=j_{0} C, k=k_{0} C$ и, вычислив порядки $|i|,|j|,|k|,|i k|,|j k|$, получаем равенство $\operatorname{Co}_{1}=\operatorname{gr}(i, j, k)$ и включение

$$
C_{2}\left(C o_{1}\right) \ni(10,10) \text {. }
$$

\section{Список литературы}

1. Горенстейн Д., Конечные простые группы. Введение в их классификачию. Мир, Москва, 1985.

2. Нерешенные вопросы теории групп. Коуровская тетрадь. Ин-т математики СО РАН, Новосибирск, 2002.

3. Нужин Я. Н., Порождающие тройки инволюций групп лиева типа над конечным полем нечетной характеристики. II. Алгебра и логика (1.997) 36, №4, 422-440.

4. Тимофеенко А. В., О порождающих тройках некоторых спорадических групп. Деп. ВИНИТИ 19.03.2001, №693-B2001.

5. Тимофеенко А. В., Порождающие тройки инволюций групп Лайонса и Янко $J_{4}$. Tезисы докл. Украинского матем. конгресса. Ин-т математики НАН Украины, Киев, 2001, с. 50.

6. Макосий А. И., Тимофеенко А. В., О порождающих группы Харады-Нортона и Фишера $F i_{24}^{\prime}$ тройках инволюций. Тезисы докл. 2-го Всесибирского конгресса женщин-математиков. Красноярский госунив., Красноярск, 2002, 133-134.

7. Каргаполов М. И., Мерзляков Ю. И., Основы теории групп. Наука, Москва, 1982.

8. Linton S. A., The maximal subgroup of the Thompson group. J. London Math. Soc. (1989) 39, №1, 79-88.

9. Linton S. A., Corrections to "The maximal subgroup of the Thompson group." J. London Math. Soc., (1991) 43, №2, 253-255. 